\title{
Experimental Verification of the Non-Stationary Statistical Model for V2V Scatter Channels
}

\author{
Michael Walter *, Uwe-Carsten Fiebig * and Alenka Zajić $\sharp$ \\ * German Aerospace Center (DLR), Münchener Straße 20, 82234 Weßling, Germany \\ $\sharp$ School of Electrical and Computer Engineering Georgia Institute of Technology, Atlanta, GA 30332 USA
}

\begin{abstract}
This paper compares our non-stationary geometricstochastic channel model for vehicle-to-vehicle scatter channels with measurement data collected in a vehicle-to-vehicle measurement campaign. The measurements were conducted on a forest road near Munich at $5.2 \mathrm{GHz}$ using a car mounted transmitter and receiver platform. The data is evaluated in terms of delay and Doppler frequency and then compared to a scaled version of the joint delay Doppler probability density function. The close agreement between the analytical and empirical data confirms the utility of our non-stationary geometric stochastic model.
\end{abstract}

\section{INTRODUCTION}

Vehicle-to-vehicle (V2V) communications systems have recently drawn great attention, because they have the potential to improve convenience and safety of automobile transportation. For example, sensor-equipped cars that communicate via wireless links and thus create ad-hoc networks, can be used to reduce traffic accidents and facilitate traffic flow. V2V communications systems also find applications in intelligent transportation systems, relay-based cellular networks, and future combat systems. The simulation and performance evaluation of $\mathrm{V} 2 \mathrm{~V}$ systems, as well as the design of future, improved systems, requires a deep understanding of the underlying propagation channels.

$\mathrm{V} 2 \mathrm{~V}$ channels have characteristics that are significantly different from the better-explored fixed-to-mobile (F2M) cellular channels [1], [2]. In V2V channels, both the transmitter (TX) and the receiver $(\mathrm{RX})$ are in motion, equipped with low elevation antennas, and surrounded by local scatterers. Furthermore, the channel characteristics of $\mathrm{V} 2 \mathrm{~V}$ channels are influenced by the properties of the environment around the communicating vehicles and by typical traffic patterns. Many V2V channel models have been proposed in the literature and are summarized in [3], [4]. However, many of these models assume that propagation occurs in a wide-sense stationary uncorrelated scattering (WSSUS) environment, which is an unrealistic assumption for V2V channels [5].

Recently, a non-stationary geometric-stochastic channel model (GSCM) has been proposed in [6] where scatterers were randomly distributed in two strips along both sides of the highway. However, no closed form expressions for the impulse response could be obtained. To overcome this shortcoming, in [7], we considered the exact geometry of the scenario and derived closed form mathematical expressions to calculate the delay-dependent Doppler spectrum. With the appropriate delay probability density function (pdf), the joint delay Doppler pdf was calculated, which is presumably proportional to the local scattering function [8].

To verify our simulation model, this paper compares the non-stationary wideband model in [7] with the measurement data collected in a vehicle-to-vehicle campaign near Munich. First, the geometric stochastic model is explained. Then, the measurement campaign and the data processing techniques used to process the measured data are presented. Finally, scaled versions of the delay Doppler pdf calculated from the model are compared with the received power in the delay Doppler domain obtained from the measured data. The close agreement between the analytical and empirical curves confirms the utility of the proposed non-stationary statistical model.

The remainder of the paper is organized as follows. For ease of reference, Section II reviews our non-stationary wideband V2V GSCM. In Section III the measurement campaign is presented. The analytical and empirical results in the delay Doppler domain are compared in Section IV. Finally, Section V provides some concluding remarks.

\section{NON-STATIONARY Statistical MOdEl FOR WIDEBAND V2V SCATTER CHANNELS}

This section briefly reviews the non-stationary statistical model for wideband V2V scatter channels proposed in [7]. Our model is based on a GSCM. Therefore, the review is split in a geometric and stochastic part.

\section{A. Geometric Part}

As the first step in obtaining the joint delay Doppler pdf, the geometry of the propagation environment is defined. The distance between the TX and RX via an arbitrary scatterer can be calculated as

$$
d(\mathbf{x}, t)=\left\|\mathbf{x}-\mathbf{x}_{\mathrm{t}}(t)\right\|+\left\|\mathbf{x}-\mathbf{x}_{\mathrm{r}}(t)\right\|
$$

with $\mathrm{x}_{\mathrm{t}}(t)=\mathrm{x}_{\mathrm{t} 0}+\mathbf{v}_{\mathrm{t}} t$ and $\mathrm{x}_{\mathrm{r}}(t)=\mathrm{x}_{\mathrm{r} 0}+\mathbf{v}_{\mathrm{r}} t$ being the motion vectors of TX and RX. The positions at $t=0 \mathrm{~s}$ are given by $\mathbf{x}_{\mathrm{t} 0}=[-d / 2,0]^{\mathrm{T}}$ and $\mathbf{x}_{\mathrm{r} 0}=[d / 2,0]^{\mathrm{T}}$ with $d$ being the line-ofsight (LOS) distance between both cars. In our model, scatterers are grouped in such a way that each group of scatterers yields the same delay at the RX. Scatterers with the same delay are located on an ellipse with TX and RX in the foci of the ellipse. The propagation delay is directly proportional to the distance via $\tau(\mathbf{x}, t)=d(\mathbf{x}, t) / c$, where $c$ is the speed of light. With the fixed delay, it is possible to evaluate the Doppler frequency for a given delay, which therefore makes it a wideband model.

The Doppler frequency $f_{\mathrm{d}}$ is a measure of the instantaneous change in distance between TX and RX via the scatterer. A positive Doppler frequency indicates a shrinking distance, whereas a negative Doppler frequency indicates a growing distance. We therefore refrain from the usual notation $f_{\mathrm{d}}=\cos (\alpha) v f_{\mathrm{c}} / c$, but 
use an expression that better describes the instantaneous change in frequency, i.e., [7]

$$
f_{\mathrm{d}}(\mathbf{x}, t)=\left(\mathbf{v}_{\mathrm{t}}^{\mathrm{T}} \nabla d_{\mathrm{t}}(\mathbf{x}, t)+\mathbf{v}_{\mathrm{r}}^{\mathrm{T}} \nabla d_{\mathrm{r}}(\mathbf{x}, t)\right) \frac{f_{\mathrm{c}}}{c}
$$

where $\nabla$ is the gradient operator.

After providing the equation for the Doppler frequency, the Doppler frequency is evaluated for a fixed delay. As stated above the scatterers of equal delay all reside on an ellipse. Using the results from [7], the Doppler frequency can be written as a function of delay as

$$
\begin{aligned}
f_{\mathrm{d}}(\phi \mid \tau) & =\frac{\left(a_{\tau} \cos \phi+d / 2\right)}{\sqrt{\left(a_{\tau} \cos \phi+d / 2\right)^{2}+\left(b_{\tau} \sin \phi\right)^{2}}} \frac{v_{\mathrm{t}}^{\mathrm{x}}}{c} f_{\mathrm{c}} \\
& +\frac{\left(a_{\tau} \cos \phi-d / 2\right)}{\sqrt{\left(a_{\tau} \cos \phi-d / 2\right)^{2}+\left(b_{\tau} \sin \phi\right)^{2}}} \frac{v_{\mathrm{r}}^{\mathrm{x}}}{c} f_{\mathrm{c}} \\
& +\frac{b_{\tau} \sin \phi}{\sqrt{\left(a_{\tau} \cos \phi+d / 2\right)^{2}+\left(b_{\tau} \sin \phi\right)^{2}}} \frac{v_{\mathrm{t}}^{\mathrm{y}}}{c} f_{\mathrm{c}} \\
& +\frac{b_{\tau} \sin \phi}{\sqrt{\left(a_{\tau} \cos \phi-d / 2\right)^{2}+\left(b_{\tau} \sin \phi\right)^{2}}} \frac{v_{\mathrm{r}}^{\mathrm{y}}}{c} f_{\mathrm{c}},
\end{aligned}
$$

Fig. 1. Transmit and receive vehicles while crew is conducting the calibration

where $\phi$ is the single remaining parameter once $\tau$ is fixed. Note that this parameter is not equal to the polar angle. The parameters $a_{\tau}$ and $b_{\tau}$ are the semi-major and semi-minor axes of the delay ellipse induced by a certain delay $\tau$.

Eq. (3) is valid, if the semi-major axis is aligned with the $x$ axis of the Cartesian coordinate system. If not, the delay ellipse has to be rotated before inserted into (2). With the delaydependent Doppler frequency in hand, the pdfs can be derived.

\section{B. Stochastic Part}

The stochastic part covers the distribution of the scatterers and how to transform it into a probability distribution of the Doppler frequency. The probability transformation is done for uniformly distributed scatterers, but can also be calculated for arbitrary probability distribution.

A uniform distribution of the scatterers does not result in a uniform distribution of the parameter $\phi$, since the length of the ellipse does not linearly grow with $\phi$. The pdf of the parameter $\phi$ can be written as [9]

$$
p(\phi \mid \tau)=\frac{\sqrt{1-\epsilon_{\tau}^{2} \cos ^{2} \phi}}{4 \int_{0}^{\frac{\pi}{2}} \sqrt{1-\epsilon_{\tau}^{2} \cos ^{2} \zeta} \mathrm{d} \zeta},
$$

where in the denominator the complete elliptic integral of the second kind can be recognized. The pdf is therefore normalized by the circumference of the ellipse.

The pdf of the Doppler frequency $p\left(f_{\mathrm{d}} \mid \tau\right)$ is then obtained using a probability transformation for the parameter $\phi$. Since the relation between $\phi$ and $f_{\mathrm{d}}$ is a many-to-one mapping, a summation over all those values is needed. The pdf of the Doppler frequency becomes

$$
p\left(f_{\mathrm{d}} \mid \tau\right)=\sum_{\phi^{\prime} \in \mathcal{F}^{-1}\left(f_{\mathrm{d}}\right)} \frac{p\left(\phi^{\prime} \mid \tau\right)}{\left|\frac{d f_{\mathrm{d}}}{d \phi^{\prime}}\right|}
$$

where $\mathcal{F}^{-1}\left(f_{\mathrm{d}}\right)$ stands for the inverse relation between the parameter $\phi$ and the Doppler frequency $f_{\mathrm{d}}$. The correct values of the parameter $\phi$ for a given Doppler frequency $f_{\mathrm{d}}^{*}$ are found procedure to synchronize the atomic clocks.

numerically by solving $f_{\mathrm{d}}^{*}-f(\phi \mid \tau)=0$ for $\phi$. The number of solutions for different Doppler frequencies can differ. For the delay distribution $p(\tau)$ a path loss model with an exponent $\gamma>2$ is assumed to obtain $p\left(\tau, f_{\mathrm{d}}\right)=p(\tau) p\left(f_{\mathrm{d}} \mid \tau\right)$.

\section{Measurement CAMpaign Description}

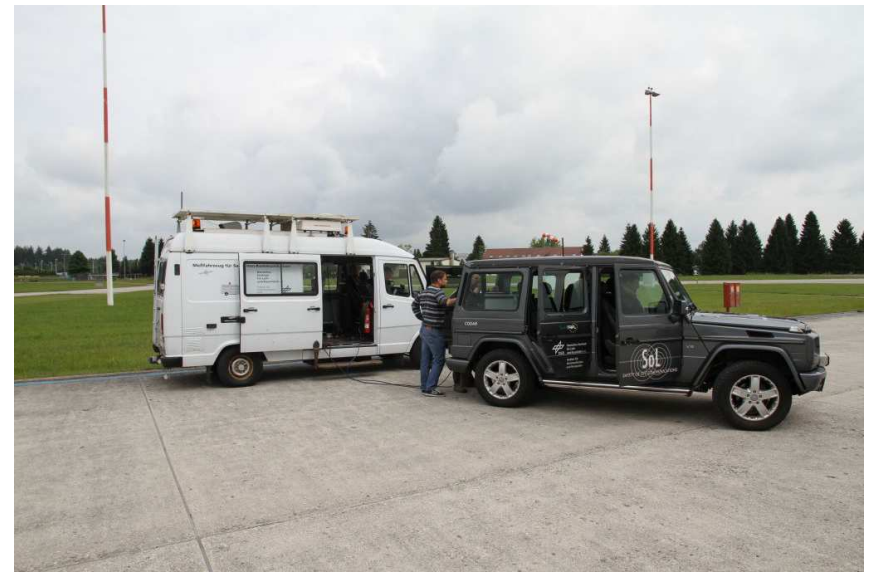

This section describes our V2V channel-sounding experimental campaign and the signal processing techniques used to process the collected data.

In order to obtain the maximum amount of scattering from the environment, a heavy forested road in the southwest of Munich was selected.

The TX was mounted onto an SUV, while the RX was put into a van, where enough space existed for the receiving equipment, see Fig. 1. The magnetic mount transmit and receive antennas were put on the roof of each car respectively. The transmit power was $P_{\mathrm{t}}=37 \mathrm{dBm}$ and omni-directional dipole antennas were used in order to receive the scattering from all directions. The antennas were suited for a frequency range of 5-6 GHz.

For the measurements, a MEDAV [10] channel sounder was used. The channel sounder transmits an OFDM-like signal and obtains the frequency response of the channel by correlating it with the known transmit sequence. The transmit frequency was $f_{\mathrm{c}}=5.2 \mathrm{GHz}$, which is very close to the future deployment frequency of $f_{\mathrm{c}}=5.9 \mathrm{GHz}$. The measurement bandwidth was $B=120 \mathrm{MHz}$ and the length of the channel impulse response (CIR) was $\tau=12.8 \mu \mathrm{s}$. The selected bandwidth leads to a delay resolution of $\Delta \tau=8.33 \mathrm{~ns}$. The relatively short length of the CIR allowed us to record with a higher cycle time compared to the maximum usable CIR length of the channel sounder. The CIR was recorded every $T_{\mathrm{c}}=1.024 \mathrm{~ms}$, which means a maximum Doppler frequency of $f_{\mathrm{d}}= \pm 488 \mathrm{~Hz}$ could be detected. This, however, is a very low Doppler frequency at this particular carrier frequency. Cars driving on a highway cause a Doppler frequency that is at least twice as high. In order not to produce alias Doppler frequencies, the speed of TX and RX had to be restricted. Therefore the vehicles only drove with a velocity of $\left\|\mathbf{v}_{\mathrm{t}}\right\|=\left\|\mathbf{v}_{\mathrm{r}}\right\|=30 \mathrm{~km} / \mathrm{h}$. Since this speed is well below the allowed speed of $\|\mathbf{v}\|=100 \mathrm{~km} / \mathrm{h}$, this further justified the selection of a less frequented road. The important measurement parameters are summarized in Table I. 
TABLE I

MEASUREMENT PARAMETERS FOR THE CAR-TO-CAR CAMPAIGN.

\begin{tabular}{ll}
\hline Center Frequency & $5.2 \mathrm{GHz}$ \\
Bandwidth & $120 \mathrm{MHz}$ \\
Signal Period & $12.8 \mu \mathrm{s}$ \\
Delay Resolution & $8.33 \mathrm{~ns}$ \\
Max. Doppler Frequency & $\pm 488 \mathrm{~Hz}$ \\
Doppler Resolution & $1 \mathrm{~Hz}$ \\
Measurement Time Grid & $1.024 \mathrm{~ms}$ \\
Tx/Rx Antenna & vehicular dipole antenna \\
Polarization & vertical \\
Transmit Power & $37 \mathrm{dBm}$ EIRP \\
\hline
\end{tabular}

In order to obtain the received power in the delay Doppler domain, the data had to be processed. Since the channel sounder records the transfer function, an IFFT is applied first to obtain the CIR. Thereafter for each block of $N_{\mathrm{b}}=1024$ CIRs an FFT was carried out to display the signal in the Doppler domain. The FFT procedure implies that the signal is averaged over $N_{\mathrm{av}}=$ $N_{\mathrm{b}} T_{\mathrm{c}}=1.05 \mathrm{~s}$. Furthermore, the synchronization between TX and RX was achieved by two Rubidium atomic clocks that were placed in each vehicle.

The locations and speeds of the vehicles were recorded with Septentrio GPS devices, which produce highly precise location data. At the RX, the GPS signal could be fed directly into the channel sounder and was saved together with the transfer function. The channel sounding data is therefore time stamped with the GPS time. With this information the corresponding location of the TX can be extracted. Furthermore, the distance between TX and RX can be determined. The width of the road was extracted from Google Earth to be $w=7.5 \mathrm{~m}$. For the scattering fields a penetration depth of $p=7.5 \mathrm{~m}$ was used, which we derived after evaluating the measurement data. The last two parameters were used as input for the geometry of the theoretic model. The vehicles are assumed to drive in the middle of the respective lane.

\section{Model VAlidation}

In this section, the scaled delay Doppler pdf obtained from the model is compared with data in the delay Doppler domain obtained from the measurement campaign described in Section III.

First, the set up for the the simulations with all the parameters is briefly described. The values for the simulation parameters are selected in such a way that they match the parameters of the measurements. In order to simplify the geometric description of the delay and Doppler frequency, the origin of the coordinate system is placed exactly in the middle between the two cars and the $x$ axis of the coordinate system is aligned with the semimajor axis of the delay ellipse. Further details on the geometry can be found in [7].

Three different scenarios are investigated:

- Scenario I: Both cars drive on the same lane in the same direction with a speed of $\mathbf{v}_{\mathrm{t}}=\mathbf{v}_{\mathrm{r}}=[30,0]^{\mathrm{T}} \mathrm{km} / \mathrm{h}$. The LOS distance is $d=40 \mathrm{~m}$. The parameters for the two scattering belts along the road are $\Delta_{1}=1.875 \mathrm{~m}, \Delta_{2}=$ $9.375 \mathrm{~m}, \Delta_{3}=5.625 \mathrm{~m}$ and $\Delta_{4}=13.125 \mathrm{~m}$, where $\Delta_{1}$ is the distance from the middle of the right lane to the beginning of the right scattering belt, $\Delta_{2}$ is the distance from the middle of the right lane to the end of the right scattering belt, $\Delta_{3}$ is the distance from the middle of the right lane to the beginning of the left scattering belt, and $\Delta_{4}$ is the distance from the middle of the right lane to the end of the left scattering belt.

- Scenario II: Both cars drive on different lanes in opposite directions approaching each other on different lanes with a speed of $\mathbf{v}_{\mathrm{t}}=[31,-0.6]^{\mathrm{T}} \mathrm{km} / \mathrm{h}$ and $\mathbf{v}_{\mathrm{r}}=$ $[-30,0.6]^{\mathrm{T}} \mathrm{km} / \mathrm{h}$. The LOS distance varies in the interval $d \in[189,204] \mathrm{m}$. The parameters for the belt are $\Delta_{1}=$ $3.75 \mathrm{~m}, \Delta_{2}=11.25 \mathrm{~m}, \Delta_{3}=3.75 \mathrm{~m}$, and $\Delta_{4}=11.25 \mathrm{~m}$, where $\Delta_{1}$ is the distance from the middle of the road to the beginning of the right scattering belt, $\Delta_{2}$ is the distance from the middle of the right lane to the end of the right scattering belt, $\Delta_{3}$ is the distance from the middle of the road to the beginning of the left scattering belt, and $\Delta_{4}$ is the distance from the middle of the right lane to the end of the left scattering belt.

- Scenario III: Both cars drive on different lanes in opposite directions receding from each other on different lanes with a speed of $\mathbf{v}_{\mathrm{t}}=[30,0.6]^{\mathrm{T}} \mathrm{km} / \mathrm{h}$ and $\mathbf{v}_{\mathrm{r}}=$ $[-28,-0.5]^{\mathrm{T}} \mathrm{km} / \mathrm{h}$. The LOS distance varies in the interval $d \in[186,203] \mathrm{m}$. The parameters for the belt are $\Delta_{1}=3.75 \mathrm{~m}, \Delta_{2}=11.25 \mathrm{~m}, \Delta_{3}=3.75 \mathrm{~m}$, and $\Delta_{4}=11.25 \mathrm{~m}$ and the definitions of the $\Delta_{i}$ are the same as in Scenario II.

A schematic of the Scenarios is shown in [7, Fig. 3(b)]. Note that as mentioned above the representation of the measurement results is in terms of the delay and Doppler frequency. That means that the received power is averaged over the interval over which the FFT is performed. For the evaluation, we used $t_{\mathrm{av}}=1.05 \mathrm{~s}$. The joint delay Doppler however can be calculated for arbitrary time instances, but for comparison reasons it is averaged over $t_{\mathrm{av}}$ and scaled afterwards.

The vector components of the velocity have to be updated accordingly, when the LOS distance changes. The starting values are provided above and those values are updated during the simulations.

To correctly model the scattering distribution in a forest, the assumption is made that the scatterers are distributed only in two belts next to the road. This is a realistic assumption since the penetration depth of the signal into the forest is finite. Furthermore, it is assumed that there is no scattering from the road. If the surface of the road is smooth, there might only be a specular reflection coming from it, which is not considered in this paper. This effect however can be easily included into our model.

For the pdfs, the lack of scattering from the road means that the support of the function will be zero for the areas where there is no scattering. Furthermore, for the pdf to integrate to one, i.e., $\int_{-\infty}^{\infty} p\left(f_{\mathrm{d}} \mid \tau\right) \mathrm{d} f_{\mathrm{d}}=1$, the areas of nonzero probability have to be re-scaled. The details of the derivation can be found in [7]. For the propagation on a forest road, the parameter $\phi$ is modeled as:

$$
p(\phi \mid \tau)= \begin{cases}\frac{\sqrt{1-\epsilon_{\tau}^{2} \cos ^{2} \phi}}{\sum_{i=1}^{4} \int_{\phi_{0 i}}^{\phi_{1 i}} \sqrt{1-\epsilon_{\tau}^{2} \cos ^{2} \zeta} d \zeta} & \phi \in \cup_{i=1}^{4}\left[\phi_{0 i}, \phi_{1 i}\right] \\ 0 & \text { elsewhere }\end{cases}
$$

where $\phi_{0 i}$ and $\phi_{1 i}$ indicate the intersection points of the ellipse with the scattering belts. The number of ellipse segments can be at most four, since there are two scattering belts. But that 


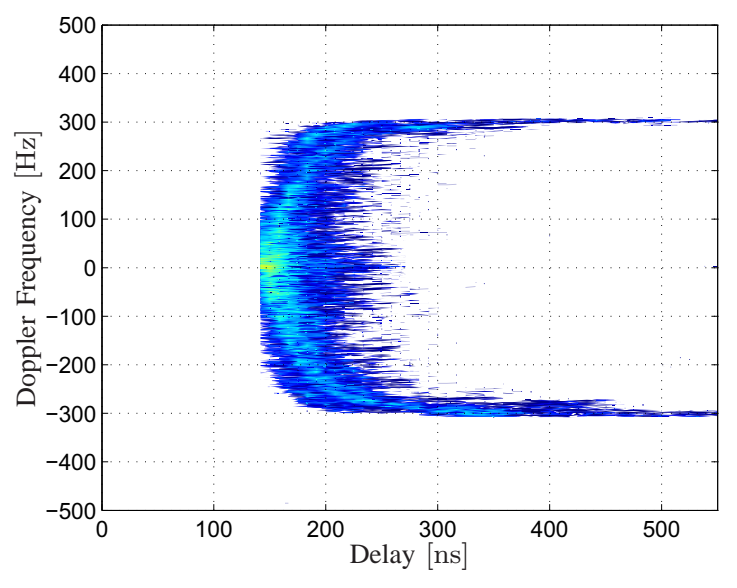

Fig. 2. Measured received power in delay Doppler domain for Scenario I.

number can be smaller, when the delay ellipses are small and do not intersect with the scattering belts [7, Fig. 3(b)].

Finally, the measurement data that we recorded during our $\mathrm{V} 2 \mathrm{~V}$ measurement campaign is presented. The received power in the delay Doppler domain is shown, since this particular presentation adequately shows the non-stationarity of the channel.

The results are then compared to a scaled version of the joint delay Doppler pdf in order to see, if our theoretic model matches the measurement data.

Since our model only takes scattering effects into account, a fair comparison only occurs, if the measurement data consists exclusively of scattering. The LOS component however was quite strong during the measurements and spilled into neighboring delay and Doppler bins. The LOS and its influence on the neighboring delays and Doppler frequencies is removed.

Fig. 2 shows the measurement results from Scenario I. At about $133 \mathrm{~ns}$ the LOS would occur, but as mentioned before, it is removed from the plot. The scattering has a U-shape in the delay Doppler domain.

The strongest scattering occurs close to the LOS. Since the scattering is confined to a scattering belt close to the road, the measurement data shows this distinctive $\mathrm{U}$ shape. For increasing delay the width of nonzero Doppler frequencies decreases. For large delays the scattering only occurs with the maximum Doppler frequency of $f_{\mathrm{d}}= \pm\left(\left\|\mathbf{v}_{\mathrm{t}}\right\|+\left\|\mathbf{v}_{\mathrm{r}}\right\|\right) f_{\mathrm{c}} / c$. Since the semi-major axis of the ellipse with the two cars in the foci is parallel to the roadside, the scattering becomes symmetric to the Doppler axis. The different colors indicate that the scattering is stronger on the inside of the $\mathrm{U}$.

The theoretical results in Fig. 3 show the same behavior for Scenario I. The theoretical scattering forms a U-shape in the Doppler delay domain and has the same width of the U-shape as the measured results. This means that the penetration depth into the forest can be determined by comparing measurement results with the theoretic pdfs. The power distribution inside the $\mathrm{U}$ can be well explained by the theoretical model. Since the cars drive on the right side of the road, different Doppler frequencies occur in the scattering belts. In the delay Doppler domain this is visible by an inner stripe that possesses higher probability for the regions where the Doppler frequency occurs in both belts

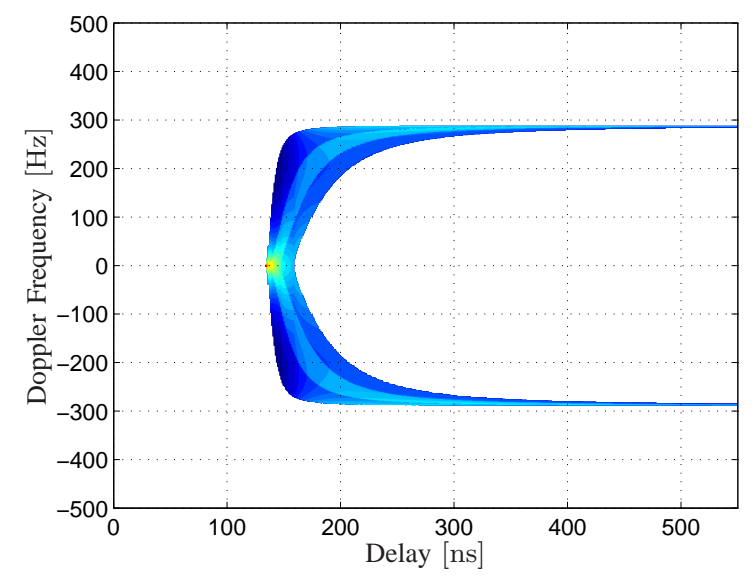

Fig. 3. Theoretical received power in delay Doppler domain for Scenario I.

and lower probability for regions where the Doppler frequency occurs only in one of the belts.

Fig. 4 shows the measurement data for Scenario II. As the cars approach each other, mainly positive Doppler frequencies can be observed. Since the geometry is changing fast, the effect of averaging becomes visible in this scenario. Thus, the scattering close to the LOS spreads in the delay direction. The power of the LOS is removed again. The Doppler frequency of the scatterers decreases fast and fades out towards two distinct values as expected at about $920 \mathrm{~ns}$.

The theoretically obtained joint delay Doppler pdf is shown in Fig. 6. The scenery and location of the scatterers within the two belts cause a wide strip in the joint pdf; for large values of the delay the pdf splits into two strips at $950 \mathrm{~ns}$. This split occurs slightly later in theory compared to the measurement data. In summary however the behavior of the theoretic pdf and the measurement data matches very well.

Fig. 5 shows the measurement data for Scenario III. The figure is almost a mirror image of Fig. 4. The scatterers close to the LOS are spread again over $90 \mathrm{~ns}$. Since the LOS distance is shorter than in Scenario II, the scattering occurs already at $600 \mathrm{~ns}$. The splitting up into two delay Doppler belts occurs at $860 \mathrm{~ns}$.

The corresponding theoretical results for Scenario III are shown in Fig. 7. As shown before, the theoretical results and the measurement data show a good resemblance. The wide strip of delay and Doppler frequencies splits into two strips at a delay of $850 \mathrm{~ns}$, which is $10 \mathrm{~ns}$ earlier as in the measurement. With the theoretical model, these two strips are easily explained. Since the scattering belts are placed symmetrically around the cars the Doppler frequencies are distributed equally in both belts. The two strips in the delay Doppler domain can therefore only result from the slightly different velocities of the cars.

\section{CONCLUSION}

In this paper we compared our non-stationary wideband channel model for V2V scatter channels with measurement data collected during a measurement campaign near Munich. The evaluation of measurement data in prior works like [11], [12] already showed promising similarities. However with our own measurements, we could exactly adjust the measurement 


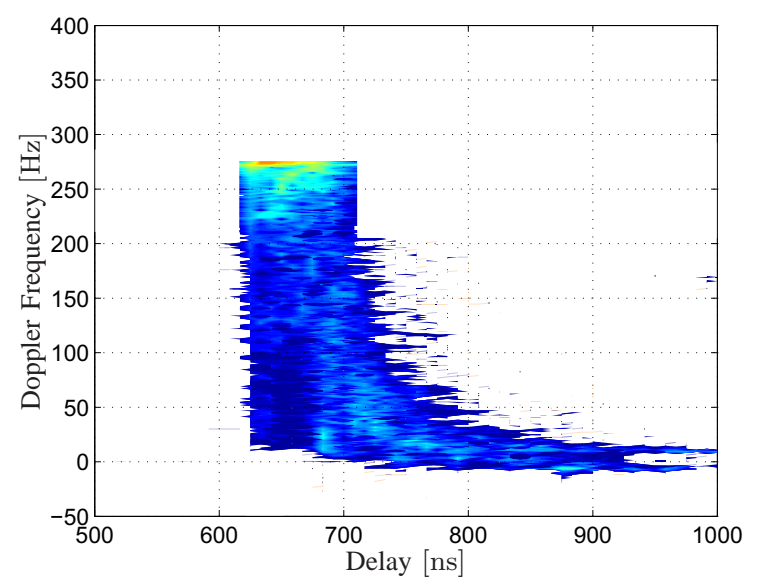

Fig. 4. Measured received power in delay Doppler domain for Scenario II.

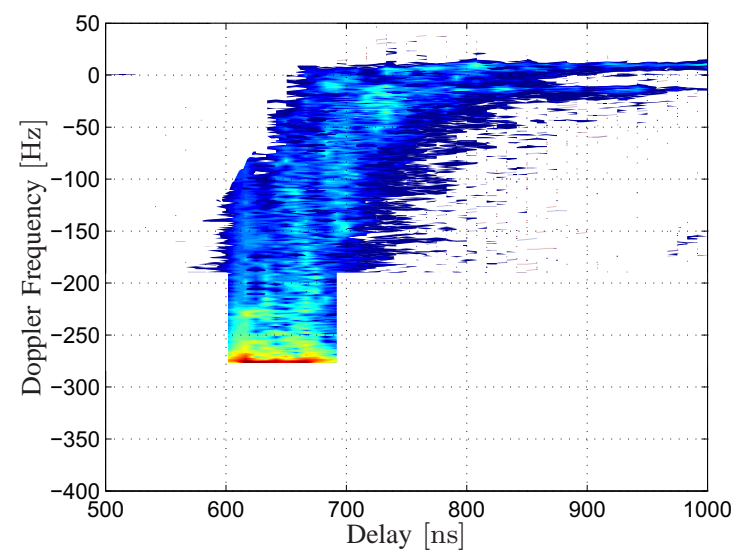

Fig. 5. Measured received power in delay Doppler domain for Scenario III.

scenarios to the theoretic models. The close agreement between the analytical and empirical results confirms the utility of our non-stationary statistical model. Additionally, both theory and measurement confirm the non-stationarity of the channel for Scenarios II and III. Depending on the scenario the channel can be stationary or non-stationary when scattering is concerned. Furthermore, we have shown that the theoretic delay Doppler pdf can be applied to simulate realistic scatter channels. The selection of the parameters can also shed light on the influence of the geometry in real world measurements. Since our GSCM is parametric, it can be used to simulate many different scattering scenarios.

\section{ACKNOWLEDGMENT}

The authors would like to thank Dmitriy Shutin, Nicolas Schneckenburger, Thomas Jost, Fabian de Ponte Müller and Christian Hauswurz for their help during the measurement campaign.

\section{REFERENCES}

[1] A. Akki and F. Haber, "A statistical model of mobile-to-mobile land communication channel," IEEE Trans. Veh. Technol., vol. 35, no. 1, pp. 2-7, Feb. 1986.

[2] A. Akki, "Statistical properties of mobile-to-mobile land communication channels," IEEE Trans. Veh. Technol., vol. 43, no. 4, pp. 826-831, Nov. 1994.

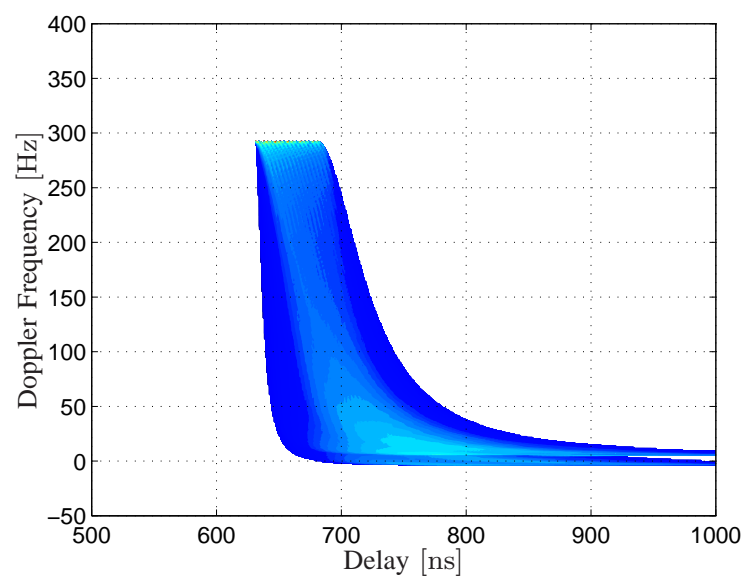

Fig. 6. Theoretical received power in delay Doppler domain for Scenario II.

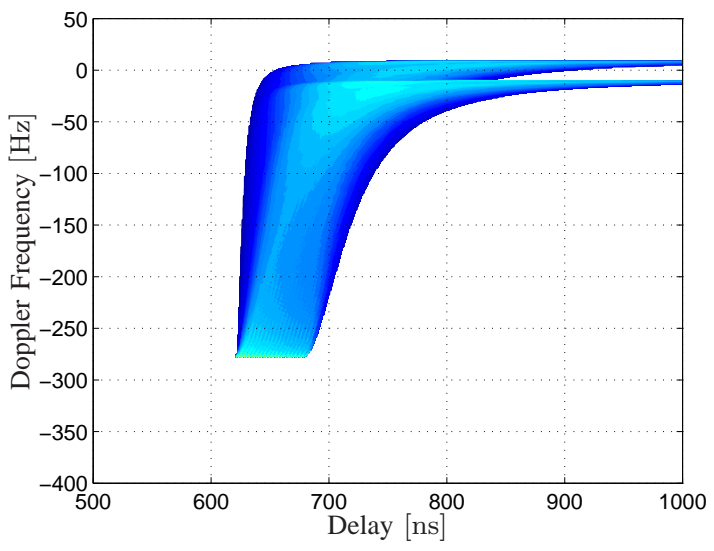

Fig. 7. Theoretical received power in delay Doppler domain for Scenario III.

[3] A. Zajić, Mobile-to-Mobile Wireless Channels, 1st ed. Boston, MA: Artech House, 2012.

[4] A. Molisch, F. Tufvesson, J. Karedal, and C. Mecklenbräuker, "A survey on vehicle-to-vehicle propagation channels," IEEE Wireless Commun., vol. 16, no. 6, pp. 12-22, Dec. 2009.

[5] A. Paier, T. Zemen, L. Bernadó, G. Matz, J. Karedal, N. Czink, C. Dumard, F. Tufvesson, A. F. Molisch, and C. F. Mecklenbräuker, "Non-WSSUS vehicular channel characterization in highway and urban scenarios at $5.2 \mathrm{GHz}$ using the local scattering function," in 2008 International ITG Workshop Smart Antennas Proc., Darmstadt, Germany, Feb. 2008, pp. 915.

[6] J. Karedal, F. Tufvesson, N. Czink, A. Paier, C. Dumard, T. Zemen, C. Mecklenbräuker, and A. Molisch, "A geometry-based stochastic MIMO model for vehicle-to-vehicle communications," IEEE Trans. Wireless Commun., vol. 8, no. 7, pp. 3646-3657, Jul. 2009.

[7] M. Walter, D. Shutin, and U.-C. Fiebig, "Delay-Dependent Doppler Probability Density Functions for Vehicle-to-Vehicle Scatter Channels," IEEE Trans. Antennas Propag., 2014, to be published.

[8] G. Matz, "On Non-WSSUS Wireless Fading Channels," IEEE Trans. Wireless Commun., vol. 4, no. 5, pp. 2465-2478, 2005.

[9] O. Nørklit and J. Andersen, "Diffuse channel model and experimental results for array antennas in mobile environments," IEEE Trans. Antennas and Propag., vol. 46, no. 6, pp. 834-840, Jun. 1998.

[10] Medav channel sounder website. [Online]. Available: http://www. channelsounder.de

[11] Y. Zhou, X. Wang, A. Guo, and F. Liu, "Channel Characterization and Simulation Modeling of Diffuse Scattering in Highway Mobile Communications," J. Inform. \& Comput. Sci., vol. 9, pp. 293-303, 2012.

[12] A. Paier, "The Vehicular Radio Channel in the 5 GHz Band," Ph.D. dissertation, Institut für Nachrichtentechnik und Hochfrequenztechnik (E389), Vienna University of Technology, 2010. 